\title{
A 3D Kinematic Model of NGC 3242
}

\author{
G. Dudziak ${ }^{1}$, J. R. Walsh ${ }^{1}$ and N. Walton ${ }^{2}$ \\ ${ }^{1}$ European Southern Observatory; ${ }^{2}$ Royal Greenwich Observatory, La Palma
}

TAURUS-II imaging Fabry-Perot data in the emission lines of $\mathrm{H} \alpha, \mathrm{He} \mathrm{II,} \mathrm{[O} \mathrm{I],} \mathrm{[O} \mathrm{III]} \mathrm{and}$ [NII] have been obtained to study the kinematics of NGC 3242. By studying the kinematic structure in lines of a range of ionization, the expansion of the nebula from close to the central star to the low ionization outer regions can be explored.

Long slit, low dispersion spectra have also been obtained and a photoionization model of the nebula was constructed. The aim of this model was primarily to obtain the emissivity as a function of radius for the different lines for which we had Taurus data cubes. A density law matching the values measured in the low ionization ([O II],[S II]) and high ionization zone ([Ar IV]) was used as a first guess, and then further refined in order to match the observed spatial variation of the $\mathrm{H} \beta$ and $\mathrm{He}$ II line profiles. The abundances were adopted from Barker(1985), hence leaving as free parameters for the modeling the ionizing central star parameters ( model atmosphere and stellar luminosity) and the filling factor $\epsilon$. A good match to the observational parameters was reach by one of our model.

We can then use the emissivity as a function of radius derived from the photoionization modeling for a range of emission lines and build model data cubes. The other input to the modellisation include the morphological structure of the nebula and its orientation relative to the observer. The velocity law followed by the gas can then be deduced from the match to the observed line profiles and to the images of the nebula in different velocity slices. A better constraint of the velocity law is achieved by using data and model cubes in different emission lines, hence probing different ionization zones in the nebula.

An equivalent narrow band $\mathrm{H} \alpha$ image of the nebula was produced by our model is in excellent agreement with the one derived from the Taurus data cube. An interesting consequence of our modeling approach is that we can get a handle on the expansion velocity of the nebula in the plane of the sky, which can be then further used in conjunction with the expansion proper motion measurements made with the VLA (Hajian et al. 1995). A first estimation of this velocity for NGC 3242 leads to a value of $20.1 \mathrm{kms}^{-1}$, the resulting dis-

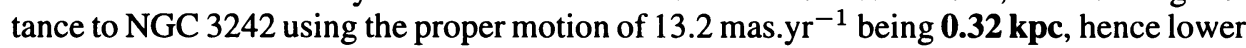
than the $0.42 \pm 0.16 \mathrm{kpc}$ distance that Hajian et al found using an expansion velocity of 26 $\mathrm{kms}^{-1}$.

\section{REFERENCES}

Barker, T., 1985, ApJ, 294, 193.

Hajian, A.,R., Terzian, Y., Bignell, C., 1995, AJ, 109, 2600. 